\title{
Long Intergenic Non-Protein Coding RNA 665 Regulates Viability, Apoptosis, and Autophagy via the MiR-186-5p/MAP4K3 Axis in Hepatocellular Carcinoma
}

\author{
Yong Shan ${ }^{1}$ and Ping $\mathrm{Li}^{2}$ \\ ${ }^{1}$ Department of General Surgery, Jinchang Central Hospital, Jinchang, Gansu; \\ ${ }^{2}$ Department of Ultrasound, Shenzhen Hospital of Southern Medical University, Shenzhen, China.
}

\begin{abstract}
Purpose: Long intergenic non-protein coding RNA 665 (LINC00665) plays a vital role in the development of cancer. Its function in hepatocellular carcinoma (HCC), however, remains largely unknown.

Materials and Methods: The expressions of LINC00665, miR-186-5p, and MAP4K3 were determined by qRT-PCR. Cell viability and apoptosis were evaluated by MTT and flow cytometry, respectively. Autophagic puncta formation was observed by fluorescence microscopy. Bioinformatics analysis, luciferase reporter assay, RNA immunoprecipitation, and RNA pulldown were performed to identify associations among LINC00665, miR-186-5p, and MAP4K3. Western blot was utilized to examine the expressions of MAP4K3, Beclin-1, and LC3. Tumor growth was evaluated in a xenograft model.

Results: Elevations in LINC00665 were observed in HCC tissues and cells. The overall survival of HCC patients with high levels of LINC00665 was shorter than those with low levels. In vitro, LINC00665 depletion inhibited viability and induced apoptosis and autophagy. miR-186-5p interacted with LINC00665 and was downregulated in HCC tissues and cells. Upregulation of miR-186$5 p$ inhibited viability and induced apoptosis and autophagy, which were attenuated by upregulation of LINC00665. MAP4K3 was found to possess binding sites with miR-186-5p and was upregulated in HCC tissues and cells. MAP4K3 depletion inhibited viability and induced apoptosis and autophagy, which were attenuated by miR-186-5p inhibitor. In vivo, miR-186-5p expression was negatively correlated with LINC00665 or MAP4K3 in HCC tissues, while LINC00665 was positively correlated with MAP4K3. LINC00665 knockdown suppressed tumor growth.

Conclusion: LINC00665 was involved in cell viability, apoptosis, and autophagy in HCC via miR-186-5p/MAP4K3 axis, which may provide a new approach for HCC treatment.
\end{abstract}

Key Words: HCC, LINC00665, miR-186-5p, MAP4K3, autophagy

\section{INTRODUCTION}

Hepatocellular carcinoma (HCC) is one of the most common

Received: November 22, 2018 Revised: March 23, 2019

Accepted: March 29, 2019

Corresponding author: Yong Shan, MD, Department of General Surgery, Jinchang

Central Hospital, No. 53, Beijing Rd, Jinchuan, Jinchang, Gansu 737100, China.

Tel: 86-0935-8814504, Fax: 86-0935-8814504, E-mail: intersection88@126.com

-The authors have no potential conflicts of interest to disclose.

(C) Copyright: Yonsei University College of Medicine 2019

This is an Open Access article distributed under the terms of the Creative Commons Attribution Non-Commercial License (https://creativecommons.org/licenses/ by-nc/4.0) which permits unrestricted non-commercial use, distribution, and reproduction in any medium, provided the original work is properly cited. malignant cancers worldwide, ${ }^{1-3}$ with a high recurrence rate and limited treatment options. ${ }^{4}$ Although great advancements have been made, ${ }^{5}$ HCC patients are still diagnosed at an advanced stage with poor prognosis. Thus, a better understanding of the pathogenesis of HCC may provide new methods for the management thereof.

Long noncoding RNAs (lncRNAs) are transcripts longer than 200 nucleotides (nts) with no protein-coding capacity. ${ }^{6}$ Emerging evidence has demonstrated that IncRNAs play important roles in HCC progression. ${ }^{7-10}$ For example, lncRNA urothelial carcinoma-associated 1 was found to be drastically increased in HCC tissues and associated with TNM stage, metastasis, and postoperative survival. ${ }^{11}$ LncRNA zinc finger antisense 1 
was shown to function as an oncogene in HCC progression by binding miR-150. ${ }^{12}$ It has been reported that long intergenic non-protein coding RNA 665 (LINC00665) may be involved in the regulation of cell cycle pathways in HCC and upregulation of LINC00665 in patients with HCC has been found to be significantly associated with poorer overall survival (OS). ${ }^{13}$ At present, the underlying molecular mechanism and functional significance of LINC00665 in HCC progression need to be further explored.

MicroRNAs (miRNAs), small noncoding RNAs of approximately 22 nucleotides, play essential roles in the development and progression of cancer. ${ }^{14} \mathrm{An}$ increasing number of reports have highlighted associations between various miRNAs and HCC development. ${ }^{15-17}$ Autophagy is a catabolic mechanism that maintains cellular homeostasis, along with formation of autophagosomes, ${ }^{18}$ and it is involved in several biological processes, including development, aging, and degeneration. ${ }^{19}$ Moreover, aberrant regulation of autophagy is related to many diseases, including cancer. ${ }^{20,21}$ It is well documented that miRNAs are involved in regulating autophagy. ${ }^{22,23}$ Previous studies have reported that miR-143 could act as a potent inhibitor of autophagy via targeting GABAA receptor associated protein like 1 (GABARAPL1) in gastric cancer cells. ${ }^{24}$ Meanwhile, the lncRNA plasmacytoma variant translocation 1 (PVT1) has been shown to act as an endogenous sponge for miR-186-5p to promote the tumorigenesis of HCC. ${ }^{25}$ However, the regulation mechanism of miR-186-5p in apoptosis and autophagy in HCC remains largely unclear.

In the current study, we explored the expression of LINC00665, miR-186-5p, and mitogen activated protein kinase kinase kinase kinase 3 (MAP4K3) in HCC tissues and cells, and analyzed correlations among them. We found that LINC00665 played an oncogene role in HCC by sponging with miR-186$5 p$ and that miR-186-5p played a tumor-suppressive role in HCC by targeting MAP4K3. Furthermore, tumor growth was also inhibited by LINC00665 depletion in vivo. In conclusion, we deemed the LINC00665/miR-186-5p/MAP4K3 axis to be involved in cell viability, apoptosis, and autophagy in HCC.

\section{MATERIALS AND METHODS}

\section{Clinical samples and cell culture}

We enrolled a total of 76 primary HCC patients who underwent radical hepatectomy from Jinchang Central Hospital (Gangsu, China) between March 2014 and October 2018. LINC00665 expression and clinicopathological features in HCC patients (76 cases) are listed in Table 1. None of the recruited patients received any treatment prior to surgery, and all provided written informed consent. Also, our study was approved by the Institutional Review Board (IRB without number) of Jinchang Central Hospital. HCC cell lines (Huh-7, HepG2, HCCLM6, MHCC-97H, and Hep3B) and the human
Table 1. Correlation between LINC00665 Expression and Clinicopathological Features of Hepatocellular Carcinoma Patients

\begin{tabular}{|c|c|c|c|c|}
\hline \multirow{2}{*}{ Clinical feature } & \multirow{2}{*}{$\mathbf{n}$} & \multicolumn{2}{|c|}{ LINC00665 } & \multirow{2}{*}{$p$ value } \\
\hline & & High & Low & \\
\hline Age & & & & 0.265 \\
\hline$\geq 60$ & 41 & 24 & 17 & \\
\hline$<60$ & 35 & 16 & 19 & \\
\hline Sex & & & & 0.424 \\
\hline Male & 51 & 32 & 19 & \\
\hline Female & 25 & 18 & 7 & \\
\hline Tumor size & & & & 0.012 \\
\hline$\geq 5 \mathrm{~cm}$ & 44 & 28 & 16 & \\
\hline$<5 \mathrm{~cm}$ & 32 & 11 & 21 & \\
\hline Hepatitis & & & & 0.109 \\
\hline Negative & 48 & 20 & 28 & \\
\hline Positive & 28 & 17 & 11 & \\
\hline Edmondson grade & & & & 0.010 \\
\hline III+IV & 47 & 32 & 15 & \\
\hline $\mid+\|$ & 29 & 11 & 18 & \\
\hline Lymph node metastasis & & & & 0.592 \\
\hline Negative & 57 & 32 & 25 & \\
\hline Positive & 19 & 12 & 7 & \\
\hline
\end{tabular}

LINC00665, long intergenic non-protein coding RNA 665.

liver cell line HL-7702 were purchased from American Tissue Culture Collection (Rockville, MD, USA). Cells were maintained in Dulbecco's Modified Eagle Medium (Thermo Fisher Scientific, Waltham, MA, USA) supplemented with $10 \%$ fetal bovine serum (Thermo Fisher Scientific). All cells were incubated at $37^{\circ} \mathrm{C}$ with $5 \% \mathrm{CO}$.

\section{Reagent and cell transfection}

MiR-186-5p mimic (miR-186-5p), mimic negative control (NC), miR-186-5p inhibitor (anti-miR-186-5p), inhibitor negative control (anti-NC), small interfering RNA LINC00665 (siLINC00665), siMAP4K3, negative control si-NC (Scramble), pcDNA3.0 (vector), and pcDNA-LINC00665 (LINC00665) were purchased from Suzhou GENEWIZ Biological Technology Co., ltd. (Suzhou, China). A biotin molecule that was covalently attached to the $3^{\prime}$ end of the mature miR-186-5p strand (3'-Biotin-miR-186-5p mimic, Bio-miR-186-5p) and negative control (3'-Biotin-miR-NC, Bio-NC) were purchased from Thermo Fisher Scientific. HepG2 and Huh-7 cells in logarithm phase were transfected with the above-mentioned plasmids or oligos using Lipofectamine 3000 (Thermo Fisher Scientific), respectively. The transfected cells were prepared for the following experiments.

\section{qRT-PCR assay}

Total RNAs were extracted from HCC tissues and corresponding adjacent normal tissues and cells using TRIzol (Thermo Fisher Scientific). MiRNAs were isolated using miRNeasy Mini Kits (Shanghai Hao Ran Biotechnology Co., Ltd., Shanghai, 
China) reversely transcribed into complementary DNA (cDNA) using TaqMan ${ }^{\circledR}$ MicroRNA Reverse Transcription kits (Biosystems, Forster City, CA, USA). To quantify mRNAs, reverse transcription was performed using Prime Script ${ }^{\mathrm{TM}} \mathrm{RT}$ reagent kits (Takara, Shiga, Japan). qPCR was carried out using the TaqMan ${ }^{\circledR}$ Universal PCR Master Mix II (Biosystems), qPCR primers were as follows: LINC00665-F, 5'-AGCACCCCTAGTGTCAGT CA-3', LINC00665-R, 5'-TGGTCTCTAGGGAGGCAGAA-3'; GAPDH-F, 5'-AGAAGGCTGGGGCTCATTTG-3', GAPDH-R, 5'-AGGGGCCATCCACAGTCTTC-3'; MAP4K3-F, 5'-GGAGC CGGGTGATTGTGA -3', MAP4K3-R, 5' -AGAAGGGAGGTG GCAAAAAT-3'; U6-F, 5'-CTCGCTTCGGCAGCACA-3', U6-R, 5'-AACGCTTCACGAATTTGCGT-3'; miR-186-5p-F, 5'-CGGC GGCAAAGAATTCTCCTT-3', miR-186-5p-R, 5'-GTGCAGGGT CCGAGGT-3'.

\section{MTT assay}

HepG2 and Huh-7 cells transfected with the above-mentioned plasmids or oligos were seeded in 96-well plates for 24,48 , and $72 \mathrm{~h}$, and $20 \mu \mathrm{L} /$ well of MTT solution $(5 \mathrm{mg} / \mathrm{mL}$, Sigma, St. Louis, MO, USA) was added and incubated for another $4 \mathrm{~h}$. Then, the supernatants were removed, and formazan crystals were solubilized in $200 \mu \mathrm{L}$ of dimethyl sulfoxide (DMSO, Sigma). The crystals were dissolved at room temperature for 10 $\mathrm{min}$. Cell viability was evaluated by measuring absorbance at $490 \mathrm{~nm}$ using a microplate reader (Bio Tek, Winooski, VT, USA).

\section{Cell apoptosis assay}

Cell apoptosis was analyzed using FITC Annexin V Apoptosis Detection Kits (BD Biosciences, Franklin Lakes, NJ, USA). HepG2 and Huh-7 cells transfected with the above-mentioned plasmids or oligos were collected. Cells were then treated with $10 \mu \mathrm{L}$ of Annexin V-FITC and $5 \mu \mathrm{L}$ of propidium iodide (PI) in the dark for $15 \mathrm{~min}$ at room temperature. Cell apoptotic rate was detected by a FACSCalibur flow cytometer with Cell Quest software (BD Biosciences).

\section{GFP-LC3 puncta assay}

HepG2 and Huh-7 cells were co-transfected with pSELECTGFP-hLC3 plasmid (Invitrogen, San Diego, CA, USA) and the above-mentioned plasmids or RNAs. $48 \mathrm{~h}$ post-transfection, cells were fixed by $4 \%$ paraformaldehyde (Sigma) and stained with 4', 6-diamidino-2-phenylindole (DAPI, $1 \mu \mathrm{g} / \mathrm{mL}$, Beyotime, Shanghai, China). The fluorescence of GFP-LC3-labelled vacuoles (autophagosomes) was observed under a fluorescence microscope (Olympus, Tokyo, Japan). The number of GFP-LC3 positive cells was counted, and the percentage of GFP-LC3 positive cells indicated the autophagosome formation.

\section{Western blot assay}

Cells treated with the above-mentioned plasmids or oligos were lysed in RIPA buffer (Beyotime). Protein lysates were quantitated using the $\mathrm{BCA}^{\mathrm{TM}}$ Protein Assay Kit (Beyotime) and then separated by SDS-PAGE (Beyotime). The proteins were transferred into polyvinylidene difluoride membranes and then blocked with $5 \%$ non-fat milk. Afterwards, membranes were incubated with primary antibody at $4^{\circ} \mathrm{C}$ overnight and subsequently incubated with secondary antibody marked by horseradish peroxidase for $1 \mathrm{~h}$. Primary antibodies, including antiMAP4K3 (1:1000, Novus Biologicals, Centennial, CO, USA), anti-GAPDH (1:2000, Abcam; Cambridge, MA, USA). Antimicrotubule-associated protein light chain 3 (LC3, 1:1000), anti-beclin-1 (1:500), and HRP linked-secondary antibody (1:2000), were purchased from Cell Signaling Technology (Danvers, MA, USA). Protein bands were visualized using an enhanced chemiluminescence solution (Beyotime).

\section{Luciferase reporter assay}

Wild-type (wt) fragments of LINC00665 or MAP4K3 harboring the miR-186-5p binding site and their mutated (mut) seed sequences were purchased from Jin Wei Zhi Biotechnology Co., Ltd. (Beijing, China) and cloned into the pMirGLO reporter vector (Promega, Madison, WI, USA), namely LINC00665-wt and LINC00665-mut and MAP4K3-wt and MAP4K3-mut, which were co-transfected with NC mimic or miR-186-5p mimic, into HepG2 and Huh-7 cells. The luciferase activity was examined using the Dual Luciferase Reporter Assay System (Promega).

RNA immunoprecipitation and RNA pulldown assays RNA immunoprecipitation (RIP) was performed using a Magna RIP RNA-Binding Protein Immunoprecipitation kit (Millipore, Billerica, MA, USA) according to the manufacturer's instructions with slight modifications. Briefly, the cells were harvested and incubated with magnetic beads and anti-Ago2 antibody (Abcam) or IgG (Abcam). The beads were washed and incubated with proteinase $\mathrm{K}$ to digest the protein at $24 \mathrm{~h}$ after incubation. The RNA was purified by the phenol-chloroform-isoamyl alcohol reagent (Sigma) and detected by reverse transcription and PCR assays. For the RNA pulldown assay, Huh-7 cells were transfected with 3'-biotinylated miR-186-5p mimic. Then, cells were lysed and incubated with streptavidin-coupled beads, forming biotin-miRNA-IncRNA complexes. RNA was isolated and detected by PCR after the complexes were digested by proteinase K. MiR-67 of Caenorhabditis elegans was used as a negative control.

\section{Tumor xenografts in vivo}

The experiments were approved by the Animal Care and Experiments Committee of Jinchang Central Hospital. The $24 \mathrm{fe}$ male BALB/c nude mice (20-22 g, 4-6 weeks) were purchased from Shanghai Laboratory Animal Center (SLAC, Shanghai, China). HepG2 and Huh-7 cells were transfected with siLINC00665 and siNC, respectively. Then, the cells were subcutaneously injected into nude mice. The tumor volume was measured and calculated by $0.5 \times$ length $\times$ width $\times$ height every 5 days, 
and mice were euthanized 30 days after cell injection. Tumor weight was measured, and qRT-PCR and Western blot were performed to detect the expression of LINC00665 and MAP4K3, respectively.

\section{Statistical analysis}

All data are presented as a mean \pm SD. All statistical analyses were evaluated using SPSS 20.0 statistical software (IBM Corp., Armonk, NY, USA) with the Student's t-test or ANOVA. A $p$ value less than 0.05 was considered to be statistically significant.

\section{RESULTS}

\section{LINC00665 expression upregulated in HCC}

In this study, we examined the expression of LINC00665 in HCC tissues and matched normal adjacent tissues. The results showed that LINC00665 was upregulated in HCC tissues, compared with matched adjacent normal tissues (Fig. 1A). Similarly, LINC00665 was also increased in HCC cells, compared to the human liver cell line HL-7702 (Fig. 1B). To investigate the effect of the increased expression of LINC00665 on the survival of patients with HCC, the cases of HCC were split into high-expression and low-expression groups. The Kaplan-
Meier method was used for statistical analysis. The results indicated that the OS of HCC patients with high LINC00665 expression was shorter than those with low LINC00665 expression (Fig. 1C). In addition, we also found that LINC00665 expression was associated with tumor size and Edmondson grade (Table 1).

\section{Downregulation of LINC00665 inhibits HCC viability and induces apoptosis and autophagy in vitro}

Given that LINC00665 expression might be associated with progression of HCC, we analyzed the effect of LINC00665 on HCC cell viability, apoptosis, and autophagy. First, we transfected siLINC00665 or siNC into HepG2 and Huh-7 cells (Fig. 2A). MTT assay demonstrated that LINC00665 knockdown inhibited the viability in HepG2 and Huh-7 cells (Fig. 2B). Moreover, the percentage of apoptotic cells was significantly increased among HepG2 and Huh-7 cells transfected with siLINC00665, compared with the scramble group (Fig. 2C). In addition, we found that LINC00665 knockdown significantly increased the ratio of LC3-II/I and expression of Beclin-1 (Fig. $2 \mathrm{E})$. Compared with control, the percentage of GFP-LC3 positive cells were significantly increased in the siLINC00665 group (Fig. 2D).

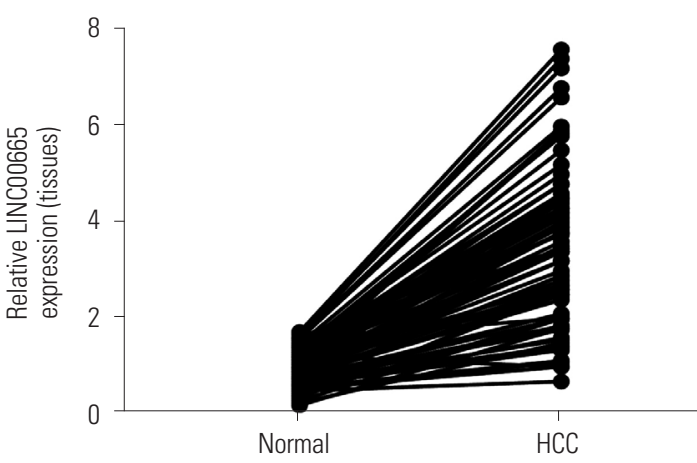

A

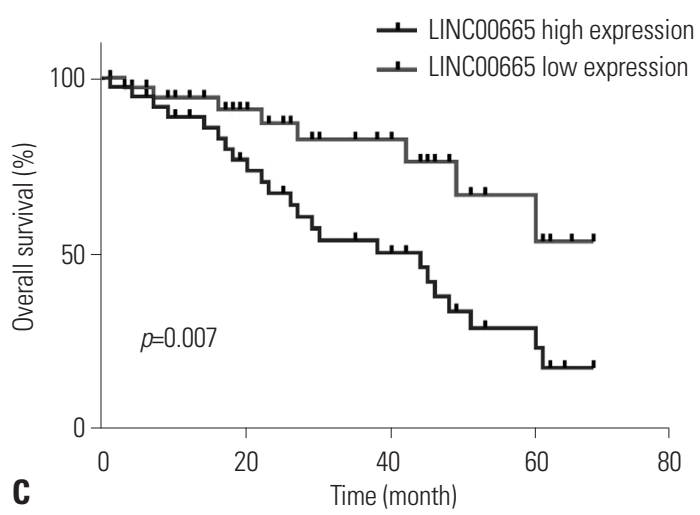

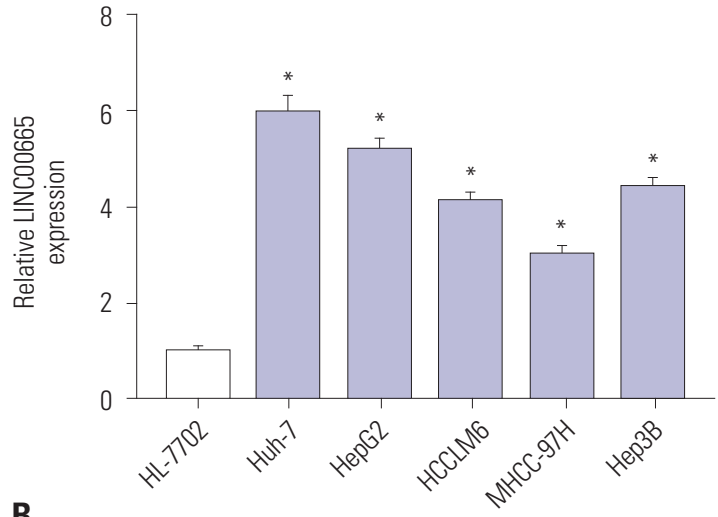

B 

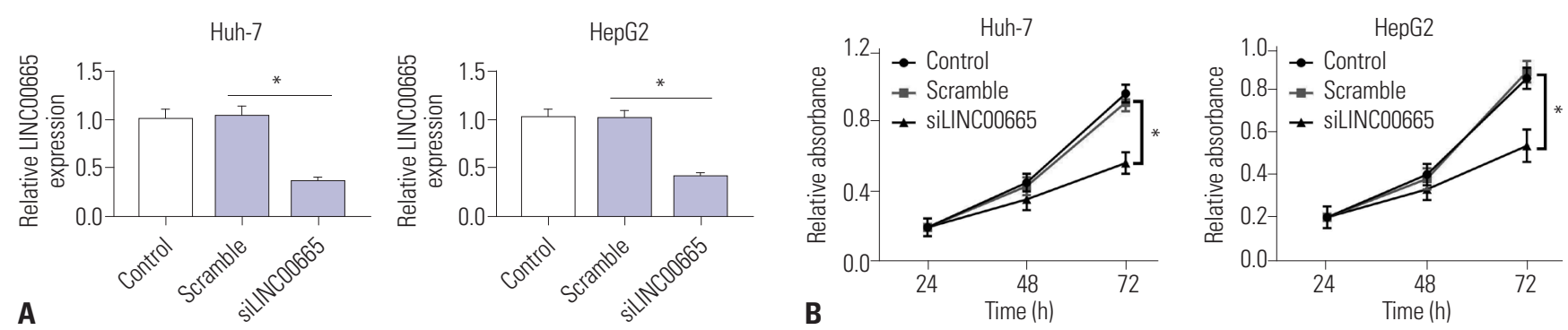
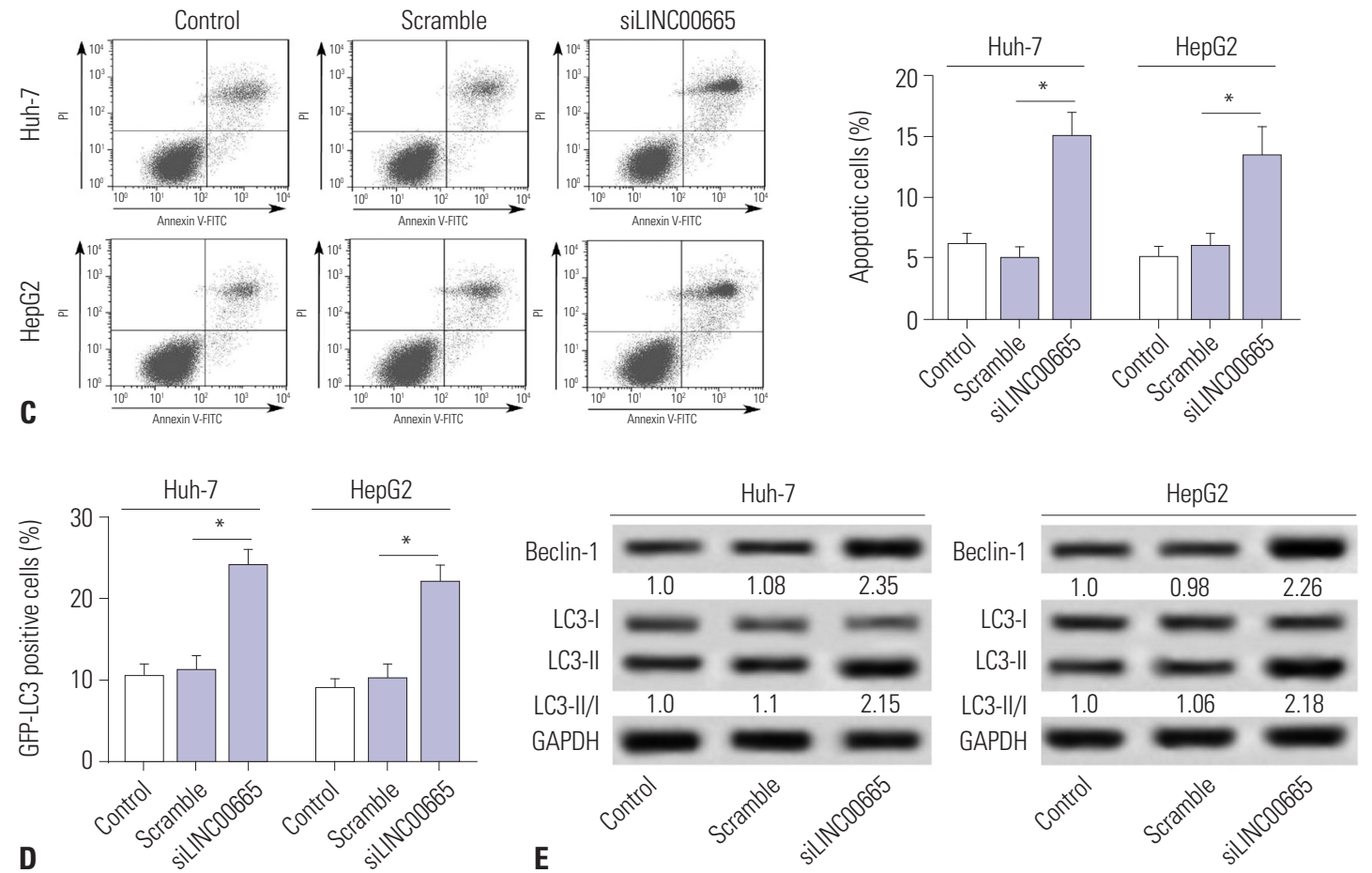

Fig. 2. Downregulation of LINC00665 inhibits hepatocellular carcinoma proliferation and induces apoptosis and autophagy in vitro. HepG2 and Huh-7 cells were transfected with siLINC00665 or siNC. (A) LINC00665 expression was detected in HepG2 and Huh-7 cells by qRT-PCR. (B) MTT assay was performed to evaluate cell proliferation. (C) Cell apoptotic rate was detected by flow cytometry. (D) Autophagy formation was analyzed by transfection of GFP-LC3 into HepG2 and Huh-7 cells. (E) Western blot was performed to detect the protein expression of Beclin- 1 and LC3. * $p<0.05$. LINC00665, long intergenic non-protein coding RNA 665.

\section{LINC00665 interacts with miR-186-5p}

To investigate the underlying molecular mechanism of LINC 00665, we predicted that LINC00665 had binding sites with miR-186-5p using starBase v2.0 (Fig. 3A). Moreover, luciferase reporter assay confirmed the relationship between LINC00665 and miR-186-5p. The results showed that miR-186-5p mimic transfection significantly decreased the luciferase activity of the LINC00665-wt group, while there was no significant impact on the luciferase activity of LINC00665-mut group (Fig. 3B). RIP and RNA pull-down assays showed that a significant amount of LINC00665 was detected, clarifying the relationship between LINC00665 and miR-186-5p (Fig. 3C and D). In addition, to further confirm the effect of LINC00665 on miR186-5p expression, the Huh-7 cells were transfected with siLINC00665, siNC, pcDNA LINC00665, or pcDNA3.0 vector. miR-186-5p was strikingly upregulated in siLINC00665-transfected Huh-7 cells and downregulated in pcDNA LINC00665- transfected cells (Fig. 3E).

\section{LINC00665 attenuates the tumor-suppressive effect of miR-186-5p on HCC cells in vitro}

To explore the expression of miR-186-5p in HCC, qRT-PCR was performed to detect the expression thereof in HCC tissues and cells. Our results demonstrated that miR-186-5p is downregulated in HCC tissues and cells, compared with matched normal adjacent tissues and the human liver cell line HL7702, respectively (Fig. 4A and B). To further investigate whether LINC00665 promotes HCC viability and blocks apoptosis and autophagy by sponging miR-186-5p, we transfected miR-NC, miR-186-5p mimic, pcDNA3.0 vector+miR-186-5p mimic, and pcDNA LINC00665+miR-186-5p mimic into Huh7 and HepG2 cells. miR-186-5p expression was significantly increased in the miR-186-5p group, which was markedly decreased in the LINC00665+miR-186-5p group (Fig. 4C). MTT 
chr19:36816453-36816474[-]

miR-186-5p 3'-tcgGGTTTTCCTCTTAAGAAAc-5'

LINC00665-wt 5'-aagCCATTAGATTAATTCTTTt-3'

A LINC00665-mut 5'-aagGGTTTTCATTTTAAGAAAt-3'
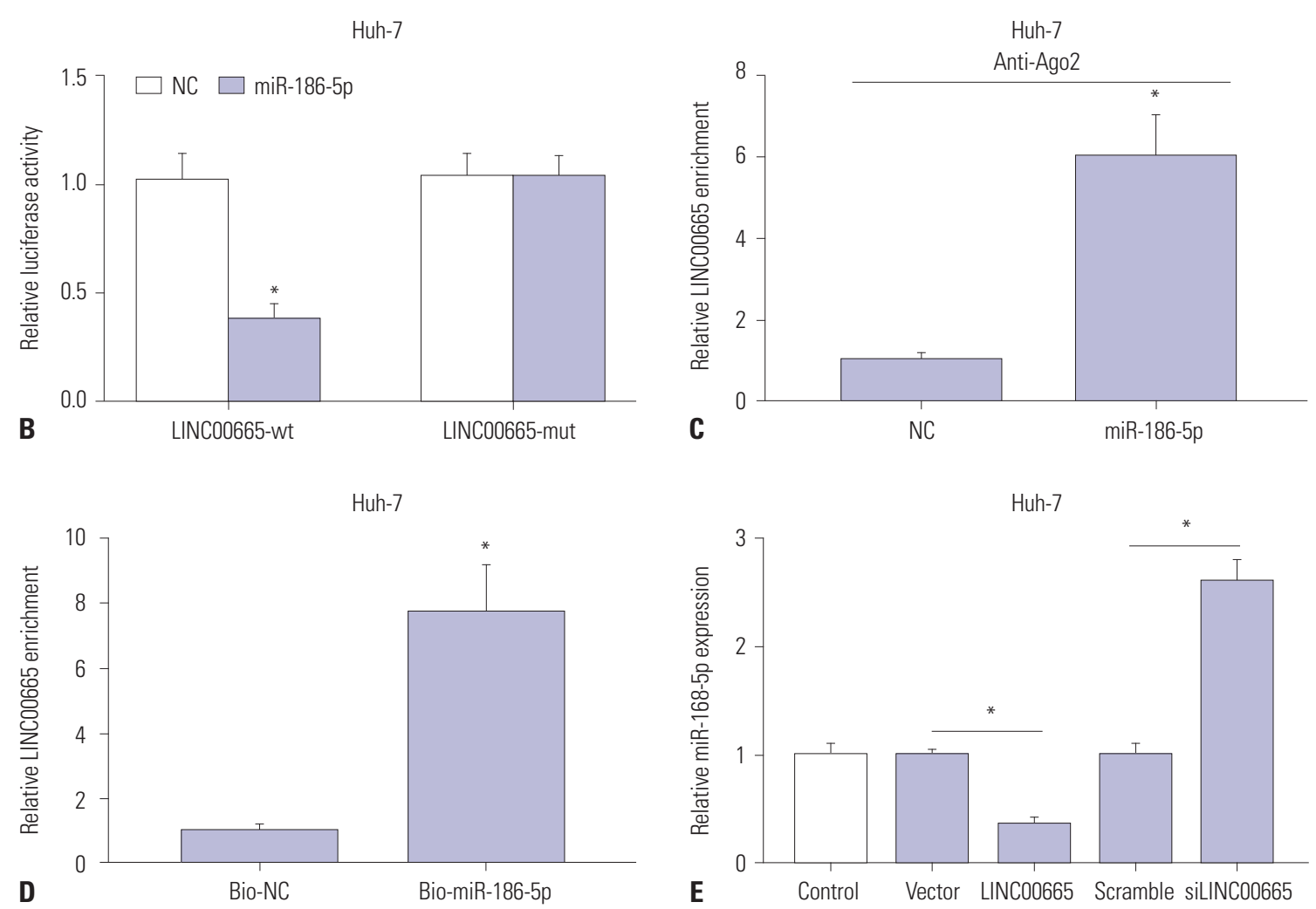

Fig. 3. LINC00665 interacts with miR-186-5p. (A) The binding sites between miR-186-5p and LINC00665 were predicted by starBase v2.0, and the luciferase reporter plasmids containing the wild-type (wt) or mutated (mut) LINC00665 binding sites of miR-186-5p were established. (B) The luciferase activity was measured in Huh-7 cells co-transfected with LINC00665-wt or LINC00665-mut luciferase reporter and miR-186-5p mimic or miR-NC. (C) The RIP assay was performed, and expression of LINC00665 was detected in samples bound to the Ago2 antibody or lgG in Huh-7 cells. (D) Detection of LINC00665 expression levels using qRT-PCR in samples pulled down by biotinylated miR-186-5p or negative control. (E) Expression levels of miR186-5p in Huh-7 cells transfected with siLINC00665, siNC, pcDNA LINC00665, or pcDNA3.0 vector. * $p<0.05$. LINC00665, long intergenic non-protein coding RNA 665.

assay demonstrated that upregulation of miR-186-5p inhibits the viability of HepG2 and Huh-7 cells (Fig. 4D). The percentage of apoptotic cells was significantly increased in HepG2 and Huh-7 cells transfected with miR-186-5p mimic, compared with the NC group (Fig. 4E). In addition, Western blot showed that upregulation of miR-186-5p significantly increased the ratio of LC3-II/I and expression of Beclin-1 (Fig. 4G). Compared with control, the percentage of GFP-LC3 positive cells were significantly increased in the miR-186-5p mimic group (Fig. 4F). Interestingly, our results showed that upregulation of LINC00665 attenuated the effect of miR-186-5p mimic on HepG2 and Huh-7 cells.

\section{MAP4K3 a target gene of miR-186-5p}

To determine whether MAP4K3 is a direct target of miR-186$5 \mathrm{p}$, TargetScan was performed to predict the binding sites between miR-186-5p and MAP4K3. As shown in Fig. 5A, miR- 186-5p has binding sites with MAP4K3. Luciferase reporter assay confirmed the relationship between MAP4K3 and miR186-5p. The results showed that miR-186-5p mimic transfection significantly decreased the luciferase activity of the MAP4K3-wt group, while there was showed no significant impact on the luciferase activity of MAP4K3-mut group (Fig. 5B). The RIP assay showed that a significant amount of MAP4K3 was detected, clarifying the direct interaction between MAP4K3 and miR186-5p (Fig. 5C). In addition, we also found that MAP4K3 was significantly downregulated in Huh-7 cells transfected with miR-186-5p mimic, but upregulated in miR-186-5p inhibitortransfected cells (Fig. 5D).

Downregulation of miR-186-5p attenuates the effect of MAP4K3 knockdown in HCC

To explore the expression of MAP4K3 in HCC, qRT-PCR was performed to detect the expression of HCC tissues and cells. 


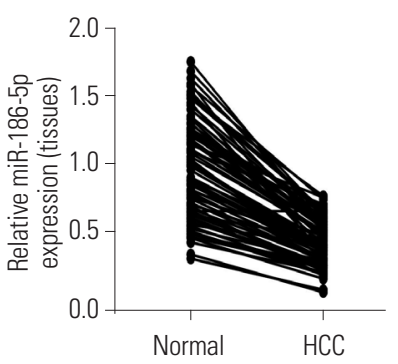

A

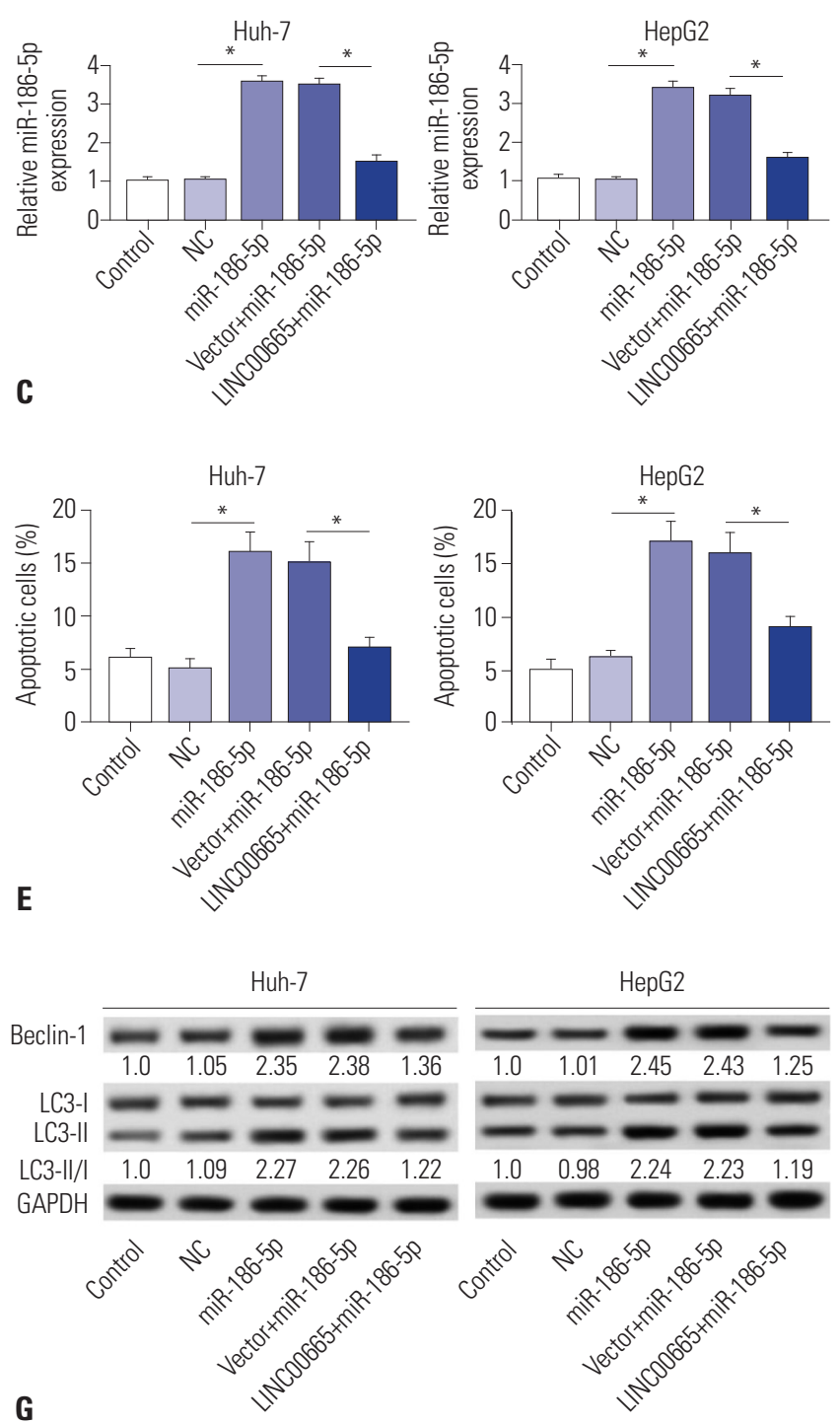

Fig. 4. LINC00665 attenuates the tumor-suppressive effect of miR-186-5p in HCC cells in vitro. (A and B) The expression levels of miR-186-5p were measured in HCC tissues and cell lines by qRT-PCR. (C-G) HepG2 and Huh-7 cells were transfected with miR-NC, miR-186-5p mimic, pcDNA3.0 vector+miR186-5p mimic, and pcDNA LINC00665+miR-186-5p mimic. (C) miR-186-5p expression was detected in HepG2 and Huh-7 cells by qRT-PCR. (D) Cell proliferation was detected by MTT assay. (E) Cell apoptotic rate was analyzed by flow cytometry. (F) Autophagy formation was analyzed by transfection of GFP-LC3 into HepG2 and Huh-7 cells. (G) Western blot was performed to detect the protein expression of Beclin-1 and LC3. ${ }^{*} p<0.05$. LINC00665, long intergenic non-protein coding RNA 665; HCC, hepatocellular carcinoma.

We discovered that MAP4K3 was increased in HCC tissues, compared with that in matched normal adjacent tissues (Fig. 6A). Similarly, it was also highly expressed in HCC cell lines (Fig. 6B). To further investigate whether miR-186-5p inhibits HCC viability and induces apoptosis and autophagy by targeting MAP4K3, we transfected si-NC, siMAP4K3, inhibitor negative control+siMAP4K3, and miR-186-5p inhibitor+siMAP4K3 into Huh-7 and HepG2 cells. MAP4K3 expression was significantly decreased in the siMAP4K3 group, but significantly increased in the anti-miR-186-5p+siMAP4K3 group (Fig. 6C). MTT assay demonstrated that MAP4K3 knockdown inhibited the viability in HepG2 and Huh-7 cells (Fig. 6D). Also, the percentage of apoptotic cells was significantly increased in HepG2 and Huh-7 cells transfected with siMAP4K3, compared with the scramble group (Fig. 6E). In addition, Western blot showed that MAP4K3 knockdown significantly increased the ratio of LC3-II/I and expression of Beclin-1 (Fig. 6G). The percentage of GFP-LC3 positive cells was markedly increased in the siMAP4K3 group, compared with control (Fig. 6F). Interestingly, our results demonstrated that cell viability was promoted and that cell apoptosis and autophagy were blocked in the antimiR-186-5p+siMAP4K3 group, compared with the siMAP4K3 group.

\section{LINC00665 regulates the expression of MAP4K3 by interacting with miR-186-5p}

As shown in Fig. 7A-C, a significant negative correlation between miR-186-5p and LINC00665 and a similar phenome- 


\section{Position 382-389 of MAP4K3-wt 3' UTR 5' ...GUUAAUAUUUUA AGAAUUCUUU A... 3' miR-186-5p 3' UCGgGUUUUCC UCUUAAGAAAC $5^{\prime}$ A MAP4K3-mut $\quad$ 3' UTR 5 '... GUUAAUAUUUUAUCUUAAGAAAA... 3'}

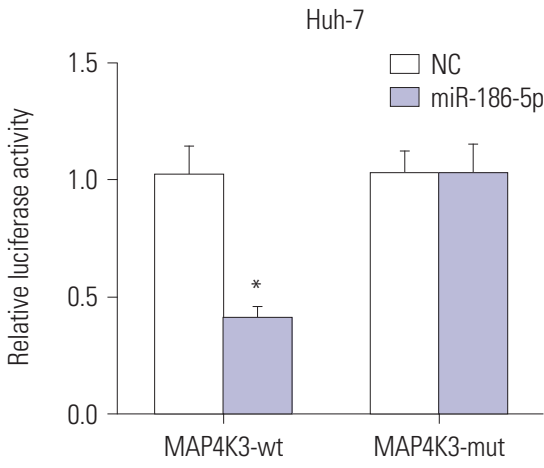

B

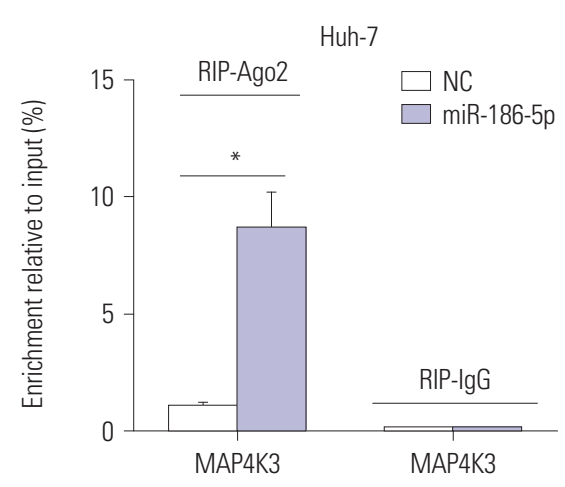

C

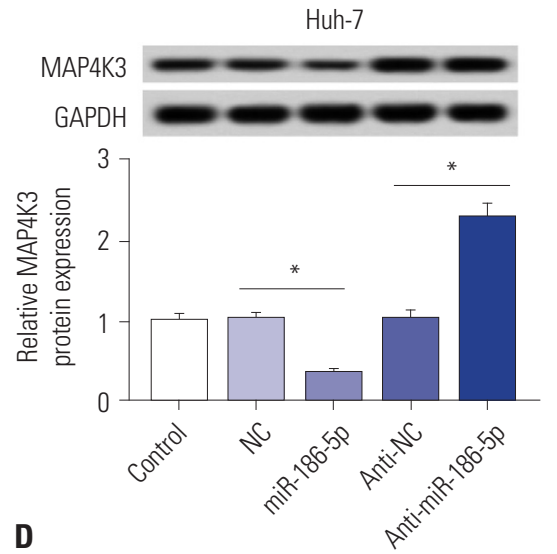

D

Fig. 5. MAP4K3 a target gene of miR-186-5p. (A) The binding sites between miR-186-5p and MAP4K3 were predicted by TargetScan, and the luciferase reporter plasmids containing the wild-type (wt) or mutated (mut) MAP4K3 binding sites of miR-186-5p were established. (B) The luciferase activity was measured in Huh-7 cells co-transfected with MAP4K3-wt or MAP4K3-mut luciferase reporter and miR-186-5p mimic or miR-NC. (C) The RIP assay was performed, and expression of MAP4K3 was detected in the samples bound to the Ago2 antibody or IgG in Huh-7 cells transfected with miR-186-5p mimic or miR-NC. (D) Detection of MAP4K3 expression level using qRT-PCR in Huh-7 cells transfected with NC mimic, miR-186-5p mimic, NC inhibitor, or miR186-5p inhibitor. * $p<0.05$. LINC00665, long intergenic non-protein coding RNA 665.

non between miR-186-5p and MAP4K3 were observed in HCC tissues. However, the expression of MAP4K3 and LINC00665 had a significant positive correlation in HCC tissues. To further understand whether LINC00665 regulates the expression of MAP4K3 by interacting with miR-186-5p, we transfected siNC, siLINC00665, inhibitor negative control+siLINC00665, or miR-186-5p inhibitor+siLINC00665 into HepG2 and Huh-7 cells. We observed that MAP4K3 expression was decreased in cells transfected with siLINC00665, which was significantly rescued by transfection with miR-186-5p inhibitor (Fig. 7D).

\section{Knockdown of LINC00665 inhibits tumor growth in vivo} To further determine the role of LINC00665 in tumorigenesis, HepG2 and Huh-7 cells transfected with siNC or siLINC00665 were injected into nude mice. The mean tumor volume in the siLINC00665 group was significantly smaller than that in the scramble group, which supported that low expression of LINC 00665 suppresses tumor growth in vivo, consistent with the in vitro results (Fig. 8A and B). qRT-PCR and Western blot assay indicated that LINC00665 and MAP4K3 expression were obviously decreased in the siLINC00665 group, compared with scramble mice (Fig. 8C and D). In addition, we also demonstrated that silencing of LINC00665 dramatically enhanced the expression of Beclin-1 and LC3, compared with that in the scramble group (Fig. 8E), which was consistent with in vitro data.

\section{DISCUSSION}

LncRNAs have been reported to act as crucial biomarkers in various cancers, including HCC. ${ }^{10,26,27}$ In this study, we found that LINC00665 was significantly upregulated in HCC tissues and cells, which was consistent with a previous report. ${ }^{13}$ Our study found that LINC00665 knockdown inhibited viability and apoptosis. Autophagy has been reported to be associated with cancer progression. ${ }^{28}$ During autophagy, the conversion of microtubule-associated protein 1 light chain 3-I (LC3-I) to LC3-phosphatidylethanolamine conjugate (LC3-II) from a cytosolic form promotes autophagosome formation. ${ }^{29}$ Beclin- 1 is a key autophagy-associated protein that strongly induces autophagy. ${ }^{29,30}$ The ratio of LC3-II/I and expression of Beclin-1 could act as indicators of autophagy. Also, an elevated number of GFP-LC3 puncta indicates enhanced formation of autophagosomes. ${ }^{31}$ In our study, we found that LINC00665 knockdown increased the GFP-LC3 positive cells, the ratio of LC3-II/I, and the expression of Beclin-1, suggesting that downregulation of LINC00665 induces autophagy.

Emerging evidence has shown that miR-186-5p plays an oncogene role in various cancers. For example, miR-186-5p expression was lower in colorectal cancer cell lines (HT116, H29, SW620, and LoVo), and upregulation of miR-186-5p inhibited viability, metastasis, and epithelial-to-mesenchymal transition in the colorectal cancer cell line LoVo. ${ }^{32}$ Also, miR-186-5p inhibitor could act as a novel strategy for targeting colorectal cancer initiation and progression. ${ }^{33} \mathrm{X}$-inactive specific transcript knockdown regulated non-small cell lung cancer prolif- 


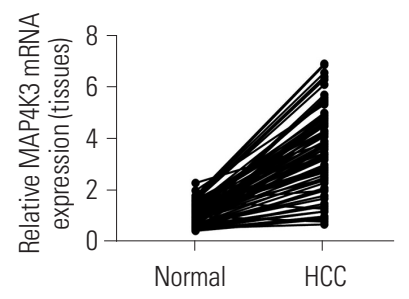

A
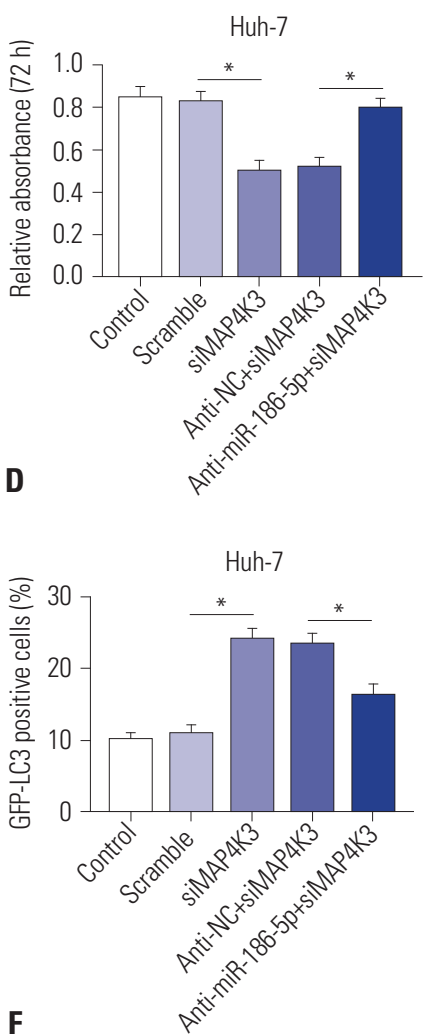

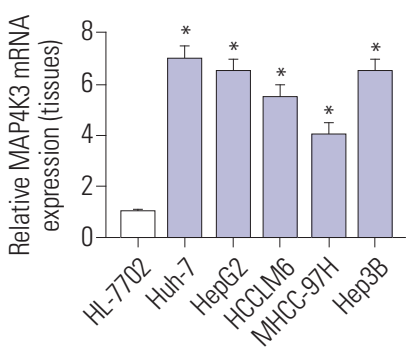

B
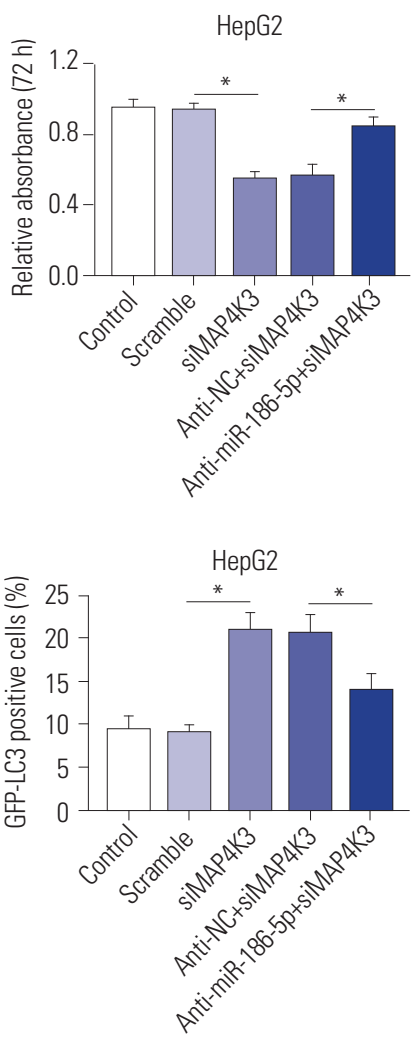
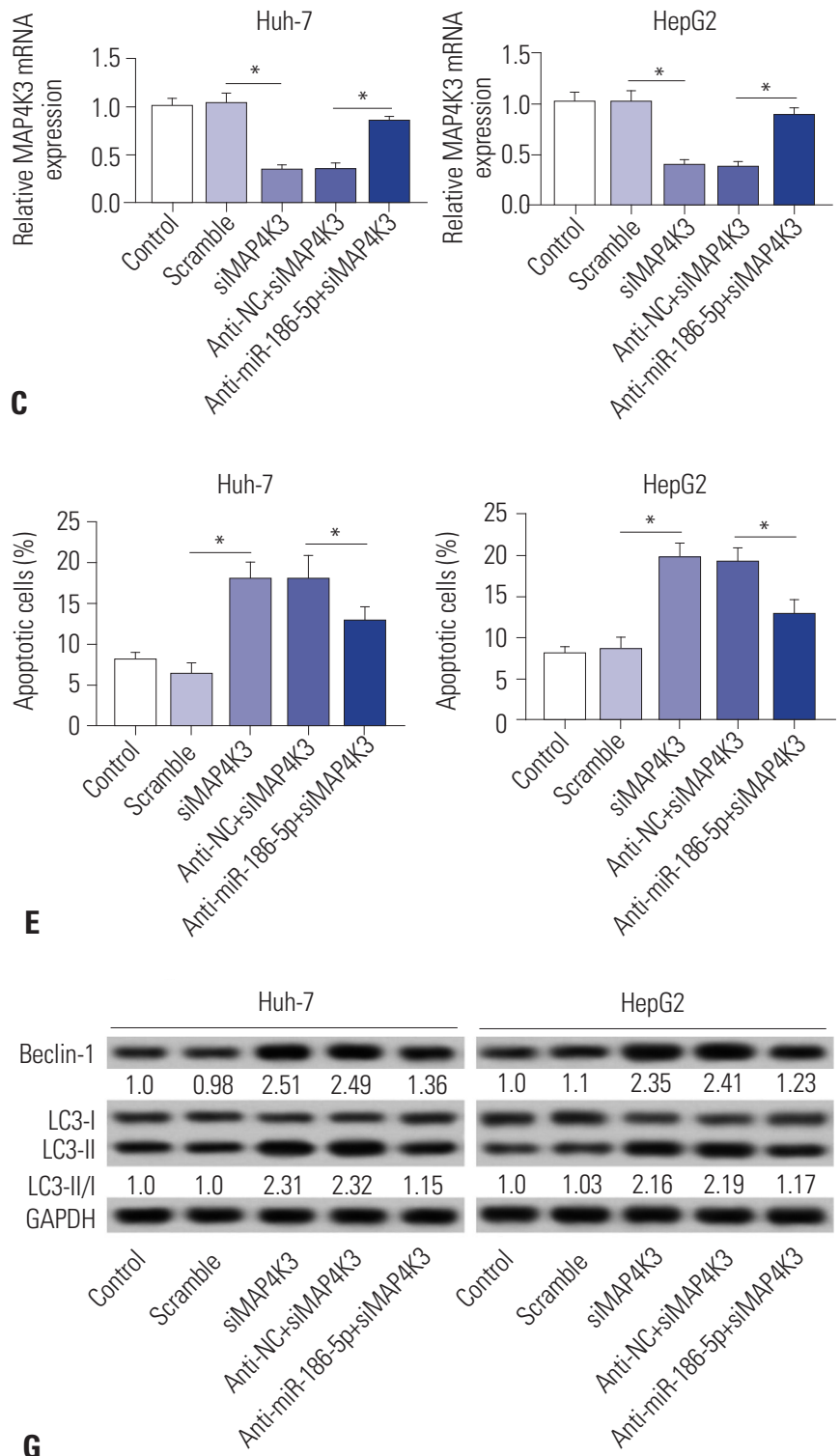

Fig. 6. Downregulation of miR-186-5p attenuates the tumor-suppressive effect of MAP4K3 knockout in HCC cells in vitro. (A and B) The expression levels of MAP4K3 were measured in HCC tissues and cell lines by qRT-PCR. (C-G) HepG2 and Huh-7 cells were transfected with si-NC, siMAP4K3, inhibitor negative control+siMAP4K3, and miR-186-5p inhibitor+siMAP4K3. (C) MAP4K3 expression was detected in HepG2 and Huh-7 cells by qRT-PCR. (D) Cell proliferation was detected by MTT assay. (E) Cell apoptotic rate was analyzed by flow cytometry. (F) Autophagy formation was analyzed by transfection of GFP-LC3 into HepG2 and Huh-7 cells. (G) Western blot was performed to detect the protein expression of Beclin- 1 and LC3. ${ }^{*} p<0.05$. HCC, hepatocellular carcinoma.

eration and invasion by modulating miR-186-5p..$^{34}$ In HCC, it also has been reported that miR-186-5p was statistically lower in HCC tissues than in normal adjacent tissues and that PVT1 promotes cell proliferation, invasion, and migration in HCC by sponging miR-186-5p. ${ }^{25}$ Interestingly, a study has indicated that miR-186-5p was elevated in prostate cancer patient serum and cell lines. ${ }^{35}$ The expression and function of miR-186-5p may be different due to differences in the microenvironments of different types of cancers. ${ }^{35}$ In the current report, we found that miR-186-5p was downregulated in HCC tissues and cells, which was consistent with previous reports. ${ }^{25}$ Furthermore, miR-186-5p expression was negatively correlated with
LINC00665 in HCC tissues. In addition, miR-186-5p exhibited binding sites with LINC00665. Luciferase reporter, RIP, and RNA pulldown assays confirmed the relationship between them. Moreover, upregulation of miR-186-5p inhibited cell viability and induced apoptosis and autophagy, suggesting miR186-5p plays a tumor suppressor role in HCC progression. Interestingly, we observed that upregulation of LINC00665 attenuates the tumor suppressing effect of miR-186-5p in HCC cells.

MAP4K3, also known as a germinal-center kinase-like kinase, is a member of the Ste20-like family of mitogen-activated protein kinases (MAPKs), which regulates diverse cellular pro- 

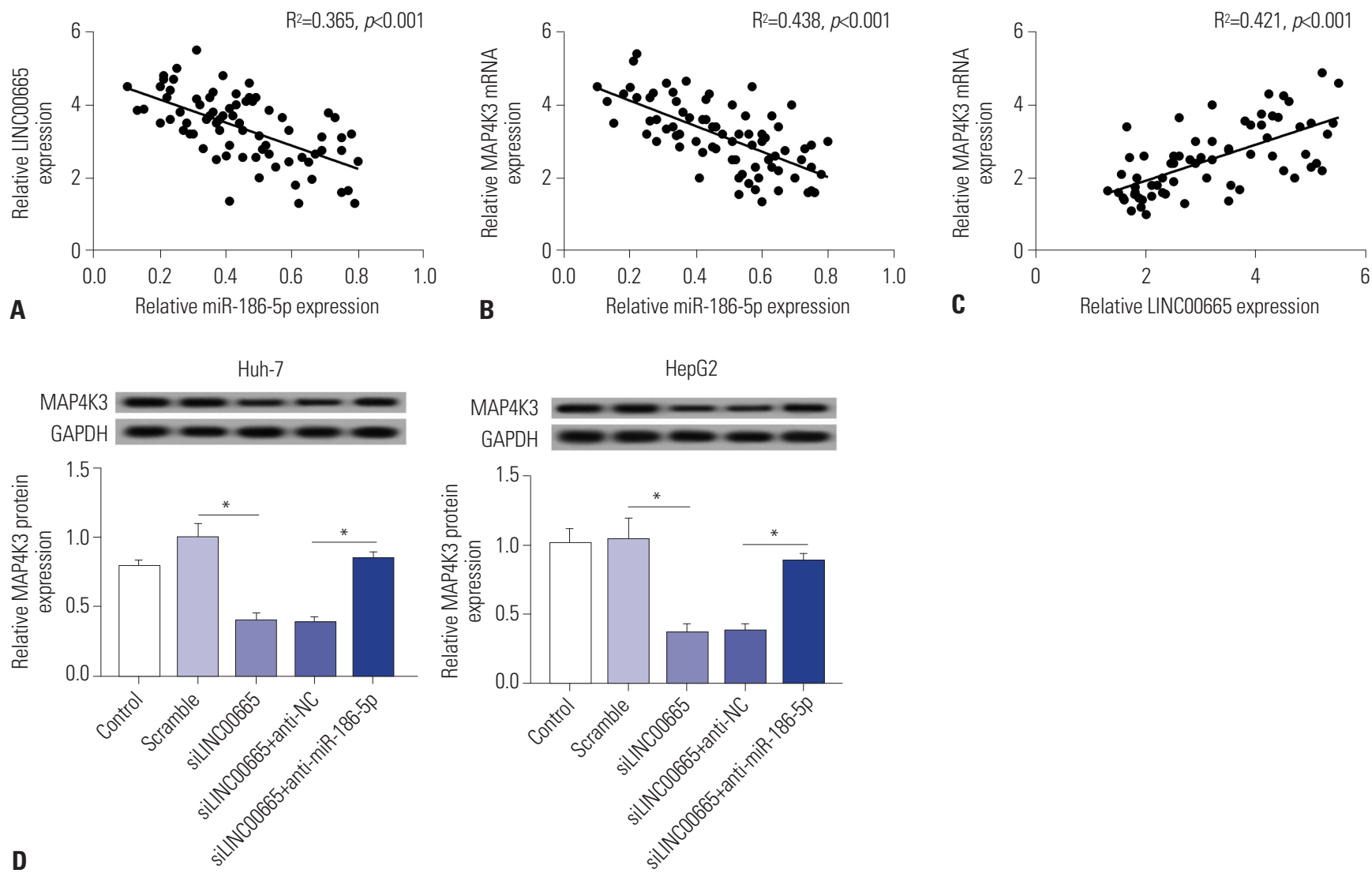

Fig. 7. LINC00665 regulates the expression of MAP4K3 by interacting with miR-186-5p. (A) The correlation between LINC00665 and miR-186-5p levels was analyzed in HCC tissues. (B) The correlation between MAP4K3 and miR-186-5p levels was analyzed in HCC tissues. (C) The correlation between LINC00665 and MAP4K3 levels was analyzed in HCC tissues. (D) Expression levels of MAP4K3 in HepG2 and Huh-7 cells transfected with siNC, siLINC00665, inhibitor negative control+ siLINC00665, and miR-186-5p inhibitor+siLINC00665. * $p<0.05$. LINC00665, long intergenic non-protein coding RNA 665; HCC, hepatocellular carcinoma.

grams by relaying extracellular signals to intracellular responses. ${ }^{36,37}$ A previous report demonstrated that miR-199a$5 p$ inhibits HCC cell migration and invasion by targeting the metastasis promoter MAP4K3 ${ }^{38}$ It also has been reported that MAP4K3 knockdown is involved in inducing productive autophagy. For example, MAP4K3 could act as an amino aciddependent regulator of autophagy through its phosphorylation of transcription factor EB. ${ }^{37}$ However, whether MAP4K3 is involved in autophagy in HCC remains unknown. In the current study, we found that miR-186-5p regulates MAP4K3 expression in Huh-7 cells. Also, MAP4K3 expression was negatively correlated with miR-186-5p in HCC tissues. In addition, MAP4K3 depletion inhibited cell viability and induced apoptosis and autophagy, suggesting that MAP4K3 may act as an oncogene in HCC progression. We also observed that downregulation of miR-186-5p attenuates the tumor suppressing effect of MAP4K3 knockdown in HCC cells. MAP4K3 expression was positively correlated with LINC00665 in HCC tissues. Notably, LINC00665 modulated the expression of MAP4K3 by sponging miR-186-5p. LINC00665/miR-186-5p/MAP4K3 signaling pathway, a new IncRNA-miRNA axis in HCC, may be involved in HCC progression by regulating cell viability, apop- tosis, and autophagy. Consistent with our in vitro results, LINC 00665 knockdown inhibited tumor growth in vivo.

However, in this research, the molecular mechanism of LINC00665 in HCC was explored based on retrospective review, which is a limitation of this work. The discovery of more new and effective genes and molecular regulatory mechanisms will be a focus in future research.

In conclusion, our data highlighted a pivotal role of LINC 00665 in the tumorigenesis of HCC. Moreover, a novel IncRNAmiRNA-mRNA regulatory network, LINC00665/miR-186-5p/ MAP4K3 axis, was first observed in HCC, which may provide a better understanding of the pathogenesis of HCC.

\section{AUTHOR CONTRIBUTIONS}

Conceptualization: Yong Shan, Ping Li. Data curation: Yong Shan, Ping Li. Formal analysis: Yong Shan. Funding acquisition: Yong Shan, Ping Li. Investigation: Yong Shan. Methodology: Yong Shan. Project administration: Yong Shan, Ping Li. Resources: Yong Shan. Software: Yong Shan, Ping Li. Supervision: Ping Li. Validation: Ping Li. Visualization: Ping Li. Writing_original draft: Ping Li, Yong Shan. Writingreview \& editing: Ping Li, Yong Shan. 
Huh-7

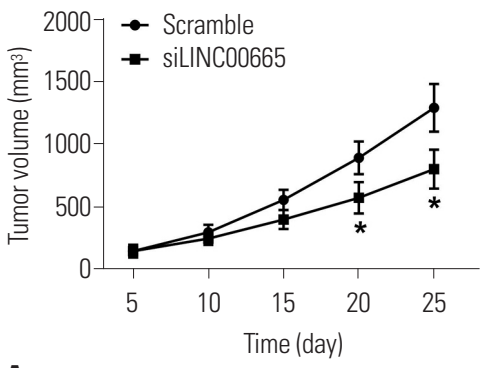

A

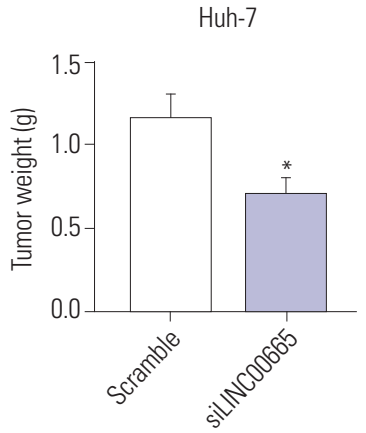

Huh-7

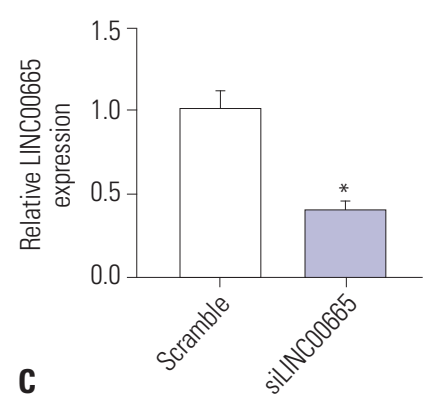

HepG2

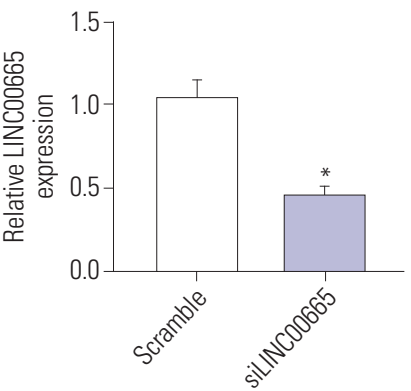

HepG2

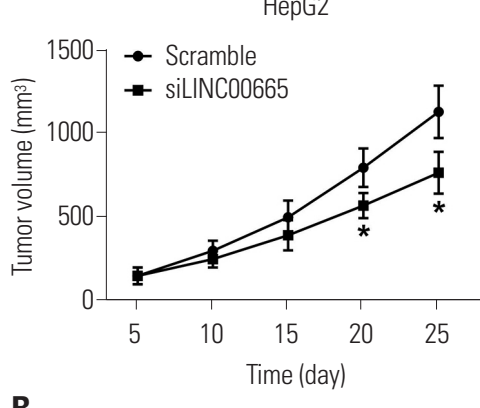

B

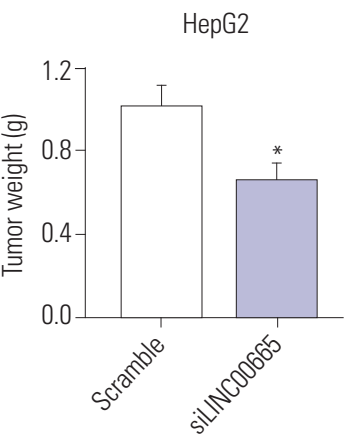

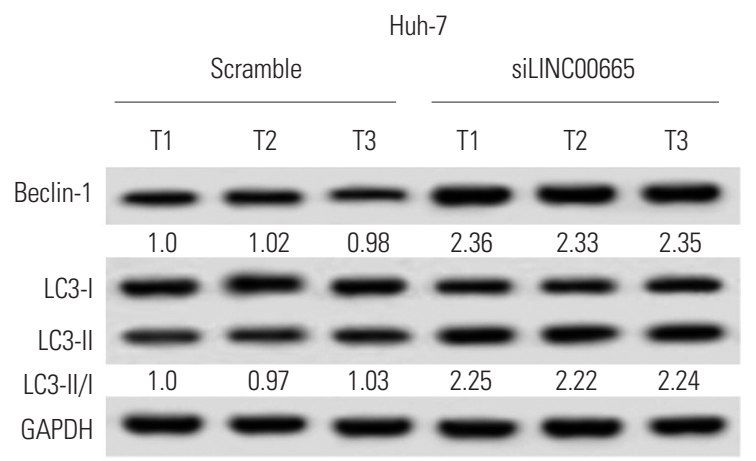

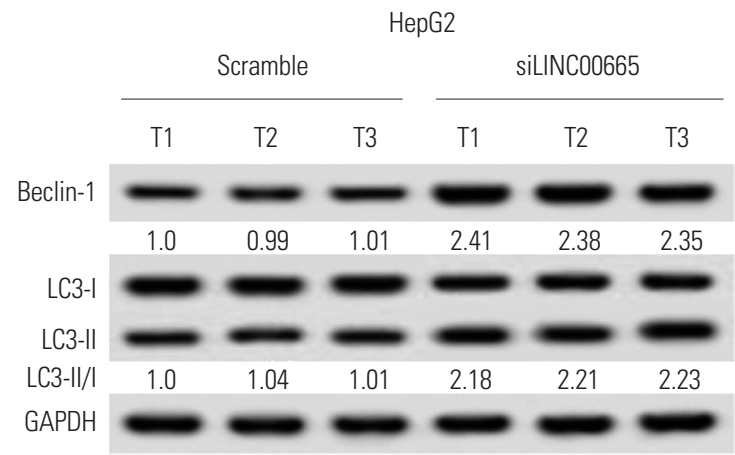

E
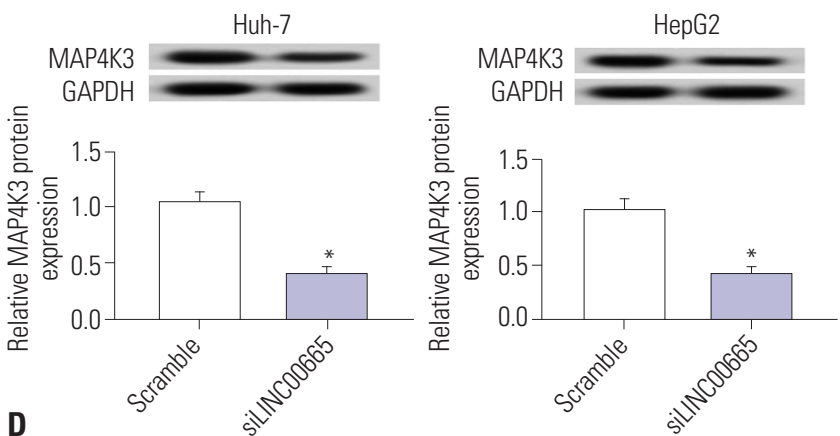

Fig. 8. Knockdown of LINC00665 inhibits tumor growth in vivo. HepG2 and Huh-7 cells transfected with siLINC00665 or siNC were subcutaneously injected into nude mice. (A and B) Tumor volume was measured at 5, 10, 15, 20, and 25 days after implantation of HepG2 or Huh-7 cells, and tumor weight was measured at 25 days after implantation of HepG2 or Huh-7 cells. (C and D) Mice were euthanized for the analysis of LINC00665 and MAP4K3 by qRT-PCR and Western blot at 25 days after implantation of HepG2 or Huh-7 cells. (E) Western blot was performed to examine the expression of Beclin-1 and LC3. ${ }^{*} p<0.05$. LINC00665, long intergenic non-protein coding RNA 665.

\section{ORCID iDs}

$\begin{array}{ll}\text { Yong Shan } & \text { https://orcid.org/0000-0002-2798-0526 } \\ \text { Ping Li } & \text { https://orcid.org/0000-0003-4661-5927 }\end{array}$

\section{REFERENCES}

1. Mak LY, Cruz-Ramón V, Chinchilla-López P, Torres HA, LoConte NK, Rice JP, et al. Global epidemiology, prevention, and management of hepatocellular carcinoma. Am Soc Clin Oncol Educ Book 2018;38:262-79.

2. Margini C, Dufour JF. The story of HCC in NAFLD: from epidemiology, across pathogenesis, to prevention and treatment. Liver Int 2016;36:317-24.

3. Yang JD, Roberts LR. Hepatocellular carcinoma: a global view. Nat
Rev Gastroenterol Hepatol 2010;7:448-58.

4. Rastogi A. Changing role of histopathology in the diagnosis and management of hepatocellular carcinoma. World J Gastroenterol 2018;24:4000-13.

5. Llovet JM, Montal R, Sia D, Finn RS. Molecular therapies and precision medicine for hepatocellular carcinoma. Nat Rev Clin Oncol 2018;15:599-616.

6. Peng JC, Shen J, Ran ZH. Transcribed ultraconserved region in human cancers. RNA Biol 2013;10:1771-7.

7. He Y, Meng XM, Huang C, Wu BM, Zhang L, Lv XW, et al. Long noncoding RNAs: novel insights into hepatocelluar carcinoma. Cancer Lett 2014;344:20-7.

8. Huang JL, Zheng L, Hu YW, Wang Q. Characteristics of long noncoding RNA and its relation to hepatocellular carcinoma. Carcinogenesis 2014;35:507-14.

9. Li G, Zhang H, Wan X, Yang X, Zhu C, Wang A, et al. Long non- 
coding RNA plays a key role in metastasis and prognosis of hepatocellular carcinoma. Biomed Res Int 2014;2014:780521.

10. Sun J, Bie B, Zhang S, Yang J, Li Z. Long non-coding RNAs: critical players in hepatocellular carcinoma. Int J Mol Sci 2014;15:2043448.

11. Wang F, Ying HQ, He BS, Pan YQ, Deng QW, Sun HL, et al. Upregulated lncRNA-UCA1 contributes to progression of hepatocellular carcinoma through inhibition of miR-216b and activation of FGFR1/ERK signaling pathway. Oncotarget 2015;6:7899-917.

12. Li T, Xie J, Shen C, Cheng D, Shi Y, Wu Z, et al. Amplification of long noncoding RNA ZFAS1 promotes metastasis in hepatocellular carcinoma. Cancer Res 2015;75:3181-91.

13. Wen DY, Lin P, Pang YY, Chen G, He Y, Dang YW, et al. Expression of the long intergenic non-protein coding RNA 665 (LINC00665) gene and the cell cycle in hepatocellular carcinoma using The Cancer Genome Atlas, the Gene Expression Omnibus, and Quantitative Real-Time Polymerase Chain Reaction. Med Sci Monit 2018; 24:2786-808.

14. Di Leva G, Garofalo M, Croce CM. MicroRNAs in cancer. Annu Rev Pathol 2014;9:287-314.

15. Song Y, Wang F, Huang Q, Cao Y, Zhao Y, Yang C. MicroRNAs contribute to hepatocellular carcinoma. Mini Rev Med Chem 2015;15: 459-66.

16. Guo W, Tan W, Liu S, Huang X, Lin J, Liang R, et al. MiR-570 inhibited the cell proliferation and invasion through directly targeting B7$\mathrm{H1}$ in hepatocellular carcinoma. Tumour Biol 2015;36:9049-57.

17. Oksuz Z, Serin MS, Kaplan E, Dogen A, Tezcan S, Aslan G, et al. Serum microRNAs; miR-30c-5p, miR-223-3p, miR-302c-3p and miR17-5p could be used as novel non-invasive biomarkers for HCVpositive cirrhosis and hepatocellular carcinoma. Mol Biol Rep 2015;42:713-20.

18. Arbogast F, Gros F. Lymphocyte autophagy in homeostasis, activation, and inflammatory diseases. Front Immunol 2018;9:1801.

19. Wang Z, Choi ME. Autophagy in kidney health and disease. Antioxid Redox Signal 2014;20:519-37.

20. Singh SS, Vats S, Chia AY, Tan TZ, Deng S, Ong MS, et al. Dual role of autophagy in hallmarks of cancer. Oncogene 2018;37:1142-58.

21. Folkerts H, Hilgendorf S, Vellenga E, Bremer E, Wiersma VR. The multifaceted role of autophagy in cancer and the microenvironment. Med Res Rev 2019;39:517-60.

22. Su Z, Yang Z, Xu Y, Chen Y, Yu Q. MicroRNAs in apoptosis, autophagy and necroptosis. Oncotarget 2015;6:8474-90.

23. Zhai H, Fesler A, Ju J. MicroRNA: a third dimension in autophagy. Cell Cycle 2013;12:246-50.

24. Du F, Feng Y, Fang J, Yang M. MicroRNA-143 enhances chemosensitivity of Quercetin through autophagy inhibition via target GABARAPL1 in gastric cancer cells. Biomed Pharmacother 2015;74: 169-77.
25. Lan T, Yan X, Li Z, Xu X, Mao Q, Ma W, et al. Long non-coding RNA PVT1 serves as a competing endogenous RNA for miR-186$5 p$ to promote the tumorigenesis and metastasis of hepatocellular carcinoma. Tumour Biol 2017;39:1010428317705338.

26. Li C, Chen J, Zhang K, Feng B, Wang R, Chen L. Progress and prospects of long noncoding RNAs (lncRNAs) in hepatocellular carcinoma. Cell Physiol Biochem 2015;36:423-34.

27. Yuan JH, Yang F, Wang F, Ma JZ, Guo YJ, Tao QF, et al. A long noncoding RNA activated by TGF- $\beta$ promotes the invasion-metastasis cascade in hepatocellular carcinoma. Cancer Cell 2014;25:666-81.

28. Huang YH, Al-Aidaroos AQ, Yuen HF, Zhang SD, Shen HM, Rozycka E, et al. A role of autophagy in PTP4A3-driven cancer progression. Autophagy 2014;10:1787-800.

29. Kang R, Zeh HJ, Lotze MT, Tang D. The Beclin 1 network regulates autophagy and apoptosis. Cell Death Differ 2011;18:571-80.

30. Qiu DM, Wang GL, Chen L, Xu YY, He S, Cao XL, et al. The expression of beclin-1, an autophagic gene, in hepatocellular carcinoma associated with clinical pathological and prognostic significance. BMC Cancer 2014;14:327.

31. Hu D, Wu J, Xu L, Zhang R, Chen L. A method for the establishment of a cell line with stable expression of the GFP-LC3 reporter protein. Mol Med Rep 2012;6:783-6.

32. Li J, Xia L, Zhou Z, Zuo Z, Xu C, Song H, et al. MiR-186-5p upregulation inhibits proliferation, metastasis and epithelial-to-mesenchymal transition of colorectal cancer cell by targeting ZEB1. Arch Biochem Biophys 2018;640:53-60.

33. Islam F, Gopalan V, Vider J, Wahab R, Ebrahimi F, Lu CT, et al. MicroRNA-186-5p overexpression modulates colon cancer growth by repressing the expression of the FAM134B tumour inhibitor. Exp Cell Res 2017;357:260-70.

34. Wang H, Shen Q, Zhang X, Yang C, Cui S, Sun Y, et al. The long noncoding RNA XIST controls non-small cell lung cancer proliferation and invasion by modulating miR-186-5p. Cell Physiol Biochem 2017;41:2221-9.

35. Jones DZ, Schmidt ML, Suman S, Hobbing KR, Barve SS, Gobejishvili L, et al. Micro-RNA-186-5p inhibition attenuates proliferation, anchorage independent growth and invasion in metastatic prostate cancer cells. BMC Cancer 2018;18:421

36. Cargnello M, Roux PP. Activation and function of the MAPKs and their substrates, the MAPK-activated protein kinases. Microbiol Mol Biol Rev 2011;75:50-83.

37. Hsu C, Lee EX, Gordon KL, Paz EA, Shen WC, Ohnishi K, et al. MAP4K3 mediates amino acid-dependent regulation of autophagy via phosphorylation of TFEB. Nat Commun 2018;9:942.

38. Liu L, Lu L, Zheng A, Xie J, Xue Q, Wang F, et al. MiR-199a-5p and let-7c cooperatively inhibit migration and invasion by targeting MAP4K3 in hepatocellular carcinoma. Oncotarget 2017;8:13666-77. 